\title{
Collagen Glycation Detected by Its Intrinsic Fluorescence
}

\author{
Rhona Muir, Shareen Forbes, David J.S. Birch, Vladislav Vyshemirsky, and Olaf J. Rolinski*
}

Cite This: J. Phys. Chem. B 2021, 125, 11058-11066

Read Online
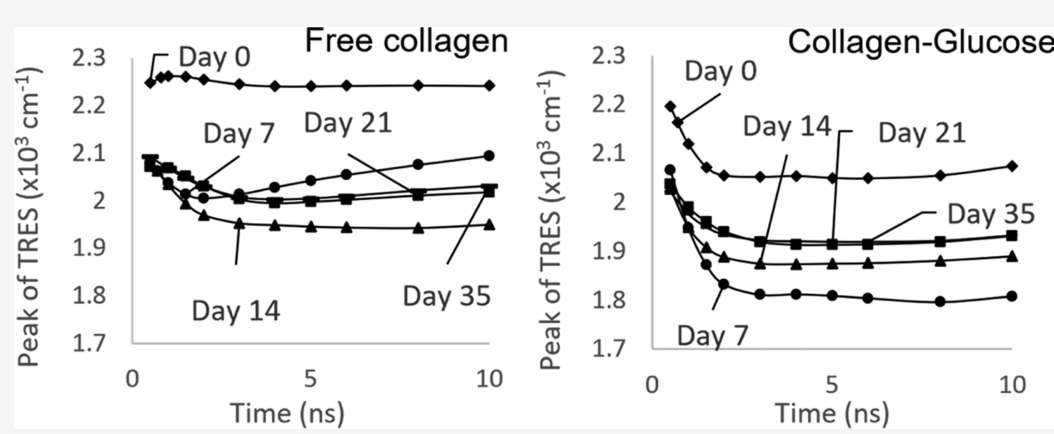

ABSTRACT: Collagen's long half-life (in skin approximately 10 years) makes this protein highly susceptible to glycation and formation of the advanced glycation end products (AGEs). Accumulation of cross-linking AGEs in the skin collagen has several detrimental effects; thus, the opportunity for non-invasive monitoring of skin glycation is essential, especially for diabetic patients. In this paper, we report using the time-resolved intrinsic fluorescence of collagen as a biomarker of its glycation. Contrary to the traditional fluorescence intensity decay measurement at the arbitrarily selected excitation and detection wavelengths, we conducted systematic wavelength- and time-resolved measurements to achieve time-resolved emission spectra. Changes in the intrinsic fluorescence kinetics, caused by both collagen aggregation and glycation, have been detected.

\section{INTRODUCTION}

Diabetes is a chronic, progressive disease characterized by elevated blood glucose levels. The increased amount of glucose in the blood associated with diabetes leads to various diabetic complications $^{1}$ such as cardiovascular and vascular diseases, stroke, kidney failure, amputation, retinopathy, and neuropathy. ${ }^{2,3}$ It has been reported ${ }^{4}$ that these complications are frequently related to the formation of compounds known as advanced glycation end products (AGEs).,

Proteins with a long biological half-life are more likely than other proteins to experience glycation and AGE formation. Collagen half-life varies between tissues, and in skin is approximately 10 years, ${ }^{5}$ which makes it highly susceptible to glycation and AGE formation. ${ }^{6}$ On collagen, glucose binds to a free lysine residue ${ }^{7}$ to form a Schiff Base and follows the classical glycation pathway. The AGEs can be in the form of adducts or cross-links within or between the proteins. ${ }^{8}$ Accumulation of cross-linking AGEs in collagen has several detrimental effects: the collagen triple helix becomes less soluble and flexible, ${ }^{5}$ reduced arterial and myocardial compliance, increased vascular stiffness, and increased diastolic function and systolic hypertension. ${ }^{9}$ Collagen glycation also augments formation and migration of myofibroblasts, which contributes to fibrosis in diabetes, ${ }^{10}$ and it is related to the duration and severity of hyperglycaemia. ${ }^{11,12}$

The risk of diabetes complications can be predicted by identifying the presence of AGEs in the dermal tissue. The studies have found ${ }^{13}$ that long-term treatment of hyperglycaemia reduced the levels of AGEs in the skin collagen. This suggests that skin AGEs, including collagen AGEs, can be used as a biomarker for diabetes complications. ${ }^{14}$

In this paper, we study the potential for using the timeresolved intrinsic fluorescence of collagen as a biomarker of its glycation. Fluorescence spectroscopy has been used extensively to study the formation and accumulation of AGEs both in vitro $^{15,16}$ and in vivo. ${ }^{14,17}$ Specifically, the use of three naturally fluorescent amino acids: phenylalanine, tryptophan, and tyrosine, ${ }^{18}$ allows the non-invasive study of biological structures and processes without the addition of any extrinsic fluorophore.

Collagen's fluorescence in the human skin ${ }^{17}$ originates from two sources: tyrosine $(\mathrm{Tyr})^{19,20}$ and collagen cross-links. As there are no tryptophan ${ }^{21}$ or phenylalanine ${ }^{22}$ residues in collagen, they do not interfere with Tyr. It can be excited at approximately $275-280 \mathrm{~nm}$ and has peak fluorescence emission at $305 \mathrm{~nm}^{20}$

Received: June 7, 2021

Revised: September 12, 2021

Published: September 24, 2021 
There are two types of collagen cross-links: ${ }^{23}$ pepsindigestible and collagenase-digestible. The former are excited at approximately $340 \mathrm{~nm}$ and emit at $400 \mathrm{~nm}$, and the latter are excited at $360 \mathrm{~nm}$ with a fluorescence emission peak at 440 $\mathrm{nm} .{ }^{17}$ Although it is not clear what structural differences there are between these two sets of cross-links, it has been confirmed experimentally $^{23}$ that fluorescence from collagen cross-links does have two distinct bands. Kollias et al. ${ }^{17}$ showed that the fluorescence intensity at $400 \mathrm{~nm}$ greatly reduced following pepsin digestion, but this had no effect on the fluorescence emission peak at $440 \mathrm{~nm}$. The opposite was true for collagenase digestion, where there was no effect on the 400 $\mathrm{nm}$ peak, but the emission at $440 \mathrm{~nm}$ almost disappeared.

AGE accumulation drives more cross-linking within collagen fibers, and so impacts its intrinsic fluorescence, ${ }^{24}$ making fluorescence emission spectra a valuable tool for monitoring AGE accumulation. ${ }^{25,26}$

Indeed, previous studies have used skin auto-fluorescence as a means of studying glycation-induced changes both in diabetic patients and healthy controls. ${ }^{25,26}$ The steady-state technique, however, is not able to fully explain the mechanisms of glycation and so help in the search for anti-glycation factors that could prevent AGE formation.

Here, we propose a more comprehensive detection of the intrinsic fluorescence by performing systematic time- and wavelength-resolved measurements, instead of performing a single measurement of the fluorescence intensity decay at the arbitrary selected excitation and detection wavelengths. Our method explores the time-resolved emission spectra (TRES) obtained at discrete times throughout the fluorescence decays. $^{27}$ Changes in TRES of the intrinsic fluorophores observed in aggregating proteins are usually complex, ${ }^{28,29}$ as they are caused by the compilation of multiple effects, mainly the heterogeneity of the sample and the dielectric relaxation of fluorophores. In these studies, we aim to verify whether the alterations in TRES triggered by collagen glycation are sufficient to help in understanding the actual mechanisms of glycation, and thus potential pathways of its prevention.

\section{MATERIALS AND METHODS}

We have studied the time-resolved responses of tyrosine (excitation at $280 \mathrm{~nm}$ ) and of pepsin-digestible cross-links (excitation at $340 \mathrm{~nm}$ ) in the collagen. Two samples were analyzed: free collagen and collagen-glucose solutions.

Tyrosine. Sample preparation followed the same protocol in both cases. Collagen type 1 solution from rat-tail and phosphate-buffered saline $\mathrm{pH} 7.4$ (PBS) were purchased from Sigma-Aldrich and used to prepare a free collagen sample of concentration $10 \mu \mathrm{M}$. The collagen-glucose sample was then prepared by adding the glucose powder (Sigma-Aldrich) to make a sample containing $10 \mu \mathrm{M}$ collagen and $20 \mathrm{mM}$ glucose. Both samples were stored in an oven at $37{ }^{\circ} \mathrm{C}$ for the duration of the experiments. All measurements were carried out using 4 $\times 1 \times 1 \mathrm{~cm}$ quartz cuvettes.

Pepsin Digestible Cross-Links. The collagen sample had a concentration of $20 \mu \mathrm{M}$, and the collagen-glucose sample contained $20 \mu \mathrm{M}$ collagen and $40 \mathrm{mM}$ glucose. Higher concentrations of both collagen and glucose were used to optimize the experiment, as the experiments at the original lower concentration showed that the fluorescence intensity at $340 \mathrm{~nm}$ excitation wavelength was too low, compromising the proper signal-to-noise ratio.
In the glucose-containing samples, the glycated collagen was not purified from the non-glycated one. The first fluorescence measurements were taken within $\sim 20 \mathrm{~min}$ of glucose being added to the sample.

Corrected fluorescence emission spectra were obtained on a Fluorolog (HORIBA Scientific) using a resolution of $1 \mathrm{~nm}$, with both the excitation and emission monochromators set to a slit width of $5 \mathrm{~nm}$. The fluorescence intensity decay measurements, and therefore, the TRES were obtained using a DeltaFlex fluorescence lifetime system (Horiba Jobin Yvon IBH Ltd, Glasgow), which uses time-correlated single photon counting (TCSPC) to record fluorescence decay. Two nanoLEDs with a repetition rate of $1 \mathrm{MHz}$ and the peak excitation wavelengths at $280 \mathrm{~nm}$ (pulse duration $<1 \mathrm{~ns}$ ) and $340 \mathrm{~nm}$ (pulse duration $<1 \mathrm{~ns}$ ) were used to study tyrosine and pepsin-digestible cross-links, respectively. To determine TRES, the fluorescence decays were recorded at a range of detection wavelengths (300-400 $\mathrm{nm}$ for Tyr, 380-500 nm for crosslinks), in increments of $10 \mathrm{~nm}$.

For both sub-experiments, the decays measured at each wavelength $\lambda$ were then fitted to the experimental fluorescence curve $I_{\exp }(\mathrm{t})$

$$
I \exp (t)=B+C_{\lambda}(t)+I_{\lambda}(t)
$$

which was assumed to be a combination of the fluorescence intensity decay $I_{\lambda}(t)$ represented by 2

$$
I_{\lambda}(t)=\sum_{i=1}^{N} \alpha_{i}(\lambda) \exp \left(\frac{-t}{\tau_{i}(\lambda)}\right)
$$

(where $\alpha_{i}(\lambda)$ and $\tau_{i}(\lambda)$ are the $i$-th pre-exponential component and its related fluorescence lifetime, respectively), $B$ which is the background fluorescence, and $C_{\lambda}(t)$ which is the scattered excitation light. Including the actual scattered excitation light $C_{\lambda}(t)$ (the fraction of the prompt function shifted in time due to the detecting photomultiplier's temporal dependence on wavelength) into the model of the experimental curve enables an accurate fluorescence decay fitting also in the case when the experimental decay has a large contribution of the scattered light. ${ }^{30}$ The Supporting Information presents the examples of fitting eq 1 and the traditional model decay (eq 2 only, with an extra exponential component with the fixed time constant equal to $1 / 2$ of the MCA time calibration value representing the scattered light) to the same decay data (see Figures S1S6). The differences in the results obtained in both approaches are mostly negligible. For TRES calculations, we used the results recovered from fitting eq 1 to the data, as the model of the measured signal assumed in this approach is more realistic.

For the data obtained for both collagen and collagen-glucose samples, a 3-exponential model $(N=3)$ was found to be sufficient to describe all fluorescence decays on the basis of the goodness-of-fit criterion $\left(\chi^{2}\right)$ and the distributions of residuals (see examples in the Supporting Information). Contrary to the common approach, however, we do not claim that this indicates 3-exponential kinetics but rather that the 3exponential function is a good mathematical representation of the observed decays.

Once values for $\alpha_{i}(\lambda)$ and $\tau_{i}(\lambda)$ were obtained, they were used to construct the TRES

$$
I_{t}(\lambda)=\frac{I_{\lambda}(t) S(\lambda)}{\sum_{i} \alpha_{i}(\lambda) \tau_{i}(\lambda)}
$$



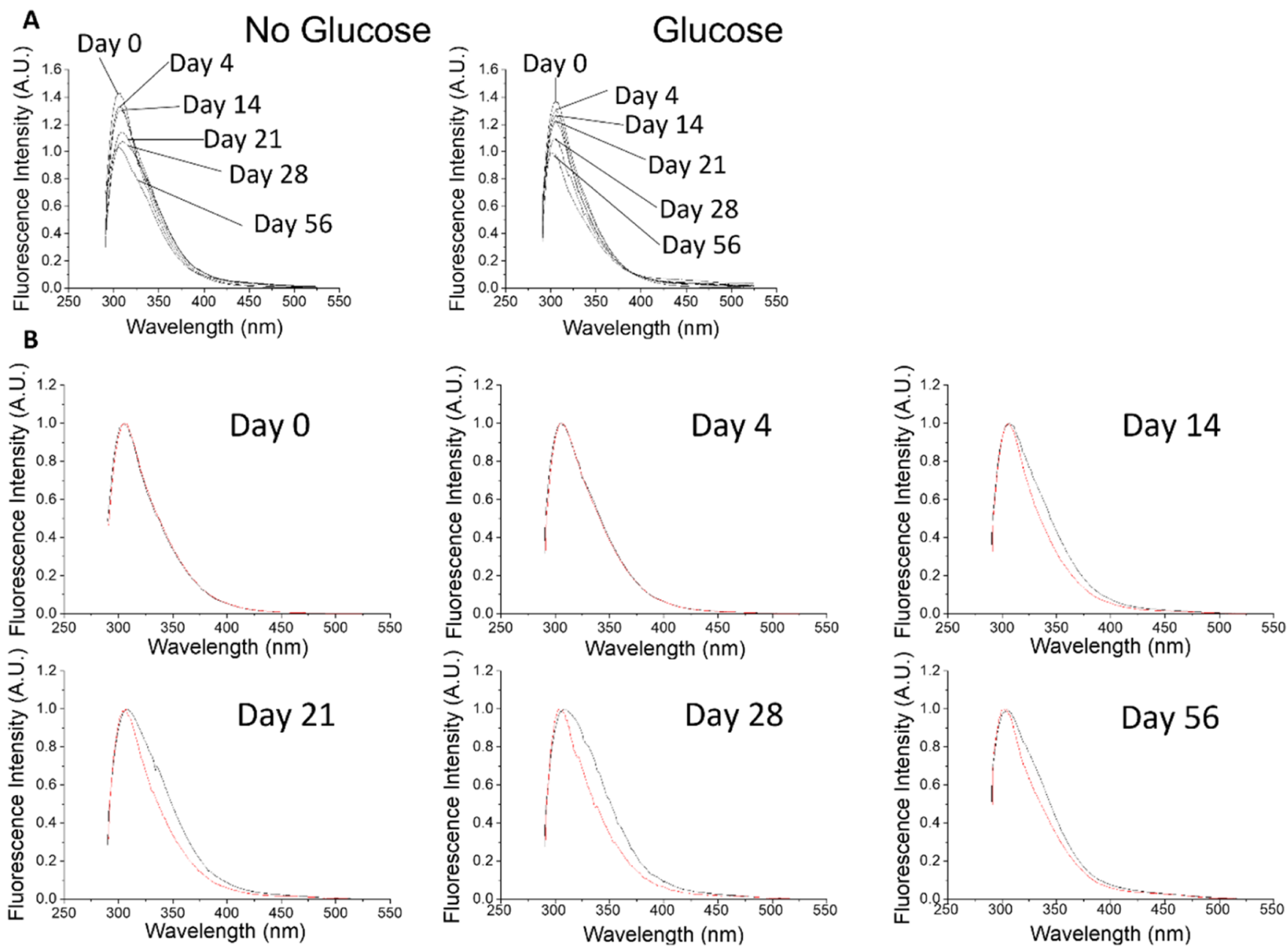

Figure 1. Fluorescence emission spectra for free collagen and collagen-glucose when excited at $280 \mathrm{~nm}$. (A) Fluorescence changes over time for collagen and collagen-glucose at days $0,4,14,21,28$, and 56. (B) Collagen (black) and collagen-glucose (red) on days 0, 4, 14, 21, 28, and 56, with both samples' spectra normalized to their peak fluorescence.

where $S(\lambda)$ is the steady-state fluorescence intensity at the emission wavelength $\lambda$ at which fluorescence decay was recorded. The TRES was finally converted to a function of wavenumber $\nu$

$$
I_{t}(\nu)=\lambda^{2} I_{t}(\lambda)
$$

This procedure generates a series of fluorescence intensity spectra as a function of wavenumber $\nu$, with each spectra representing fluorescence at a different time $t$ after excitation.

The next stage involved fitting a "technical" model of TRES to the experimental TRES, namely, we used the modified multiple Toptygin-type distribution ${ }^{31}$ to represent the shape of spectral components.

$$
\begin{aligned}
F(\mathrm{v})= & \sum_{i=1}^{M} \frac{C_{i}(t)}{\sqrt{2 \pi} \sigma_{i}(t) v_{i}(t)\left(v_{i}(t)^{2}+3 \sigma_{i}(t)^{2}\right)} v^{3} \\
& \exp \left(\frac{-\left(v-v_{i}(t)\right)^{2}}{2 \sigma_{i}(t)^{2}}\right)
\end{aligned}
$$

In this model, $M$ is the number of spectral components, $C_{i}$ is the contribution of each component to fluorescence, $v_{i}$ is the peak position, and $\sigma_{i}$ is the half-width of the distribution. The $1 /\left(\sqrt{2 \pi} \sigma_{i}(t) v_{i}(t)\left(v_{i}(t)^{2}+3 \sigma_{i}(t)^{2}\right)\right)$ term is the normalization factor for this equation. Note, that the Franck-Condon factor envelope $^{31}$ is approximated here by a Gaussian function instead of a more realistic skewed function. This allows a reduction in the number of model 5 parameters, which was necessary because of the limited number of experimental TRES points calculated in TCSPC analysis. The model function 5 was fitted to the experimental TRES points, and the potential mechanisms of the observed changes in TRES were discussed on the basis of the goodness of fit obtained for different $M$, and the changes in $v_{i}(t), C_{i}(t)$, and $\sigma_{i}(t)$ values. The experimental TRES points and the corresponding model TRES curves were found for day 0 , and for various subsequent days after preparation. The examples of the obtained plots are shown in the Supporting Information (Figures S7 and S8).

\section{RESULTS AND DISCUSSION}

Tyrosine Responses (Excitation $280 \mathrm{~nm}$ ). Figure 1 shows the fluorescence emission spectra for free collagen and collagen-glucose over 56 days, where the samples were excited at $280 \mathrm{~nm}$ to observe the response of tyrosine. The absolute fluorescence intensities of both collagen and collagen-glucose decrease over the 56 days, as shown in Figure 1A.

Changes observed in both samples could be due to aggregation and/or glycation, as both processes may affect the conformation of the protein, including the local micro- 
environment of tyrosine. Figure $1 \mathrm{~B}$ presents the pairs of spectra at individual days normalized to 1 . The narrower spectra observed in the collagen-glucose sample may be a result of glycation, where the presence of the collagen-glucose aggregates reduces the formation of bigger collagen aggregates and consequently reduces the broadening of the spectrum reflecting the narrower range of the $\pi$ electron spatial distribution. This hypothesis is further supported by TRES results discussed in the later section. It should be noted that we do not consider the potential degrading effects of UV illumination on the sample, as the intensities of light used in our measurements are minimal.

Additional information has been extracted from the analysis of TRES of both samples. This was implemented through a series of fluorescence intensity decays recorded when exciting the collagen's Tyr at $280 \mathrm{~nm}$. The decays were detected at the range $300-400 \mathrm{~nm}$ in increments of $10 \mathrm{~nm}$ (as in the example for day 0 shown in Figure 2). Figure 2 demonstrates a strong
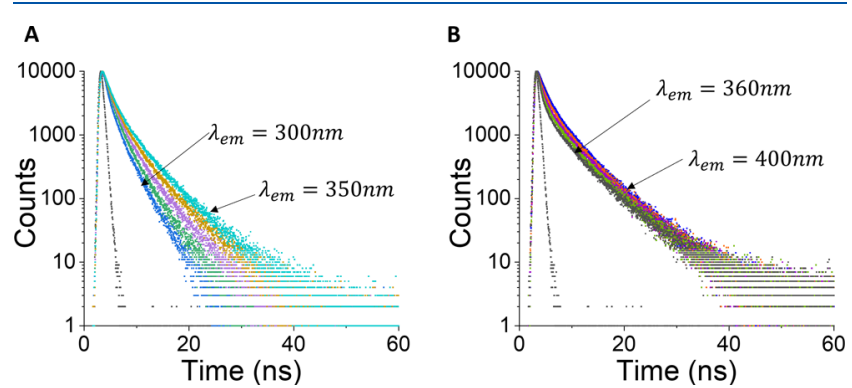

Figure 2. Fluorescence intensity decays for the collagen on day 0 , excited at $279 \mathrm{~nm}$. Part A shows the emission wavelengths from 300$350 \mathrm{~nm}$ in increments of $10 \mathrm{~nm}$ and part $B$ shows the emission wavelengths from 360-400 $\mathrm{nm}$. Both plots are shown with the prompt signal (black).

lifetime-wavelength dependence, where fluorescence at the shorter detection wavelengths decays more rapidly than at the longer wavelengths. This is consistent with the lifetimewavelength correlation observed in proteins ${ }^{32}$ and is usually caused by dielectric relaxation of intrinsic fluorophores.
The decays recorded at various times after sample preparation were used to calculate the TRES for both collagen and collagen-glucose samples. These plots, along with their related normalization plots, are shown in Figure 3. Part A (absolute spectra) and $\mathrm{E}$ (normalized spectra) show the result obtained for both samples on day 0 , where no effect of glycation is expected yet. In this case, the spectra for both the free collagen and collagen-glucose samples are very similar, with the intensity gradually decreasing within nanoseconds. This similarity demonstrates the robustness of the experimental and data processing procedures that were used for TRES calculation.

At the later times after sample preparation, differences in the TRES of both samples are observed, indicating changes in the evolution of collagen spectra caused by the presence of glucose. To obtain more quantitative information on the detected effects, the technical model of TRES (eq 5) was fitted to experimental TRES data. We used the Akaike information criterion $^{33}$ (for definition see the Supporting Information) to optimize the number $M$ of the components of the eq 5 . It has been found (see Table T1 in the Supporting Information) that to avoid over parametrization of the model, only one component $(M=1)$ should be used. Note that such a result does not imply homogeneous dielectric relaxation because the same shapes of the normalized TRES are not observed here. The evolution of the recovered $v(t), \log (C(t))$, and $\sigma(t)$ values are plotted in Figure 4.

Initially, at day 0 , the peak position of the emitting fluorophore $v(t)$ is $\sim 31,500 \mathrm{~cm}^{-1}$ for both samples. Over the 10 ns following excitation, the peaks show a shift to longer wavelengths, ending at $\sim 29,200 \mathrm{~cm}^{-1}$. We note that at day 0 there was no difference between the spectral shifts in the free collagen and glucose-collagen samples (see Figure 4G). Over the 56 days, however, the initial peak position $\nu_{\text {peak }}(t)$ of the un-glycated sample (Figure 4A) steadily decreased, still following the same pattern of a spectral shift toward longer wavelengths, thus suggesting collagen aggregation. On the other hand, the $\nu(t)$ in the glycated sample (Figure 4D) remained similar over the 56 days. This indicates that the aggregation of collagen is hindered by the glycation process. This observation is consistent with the narrower steady-state
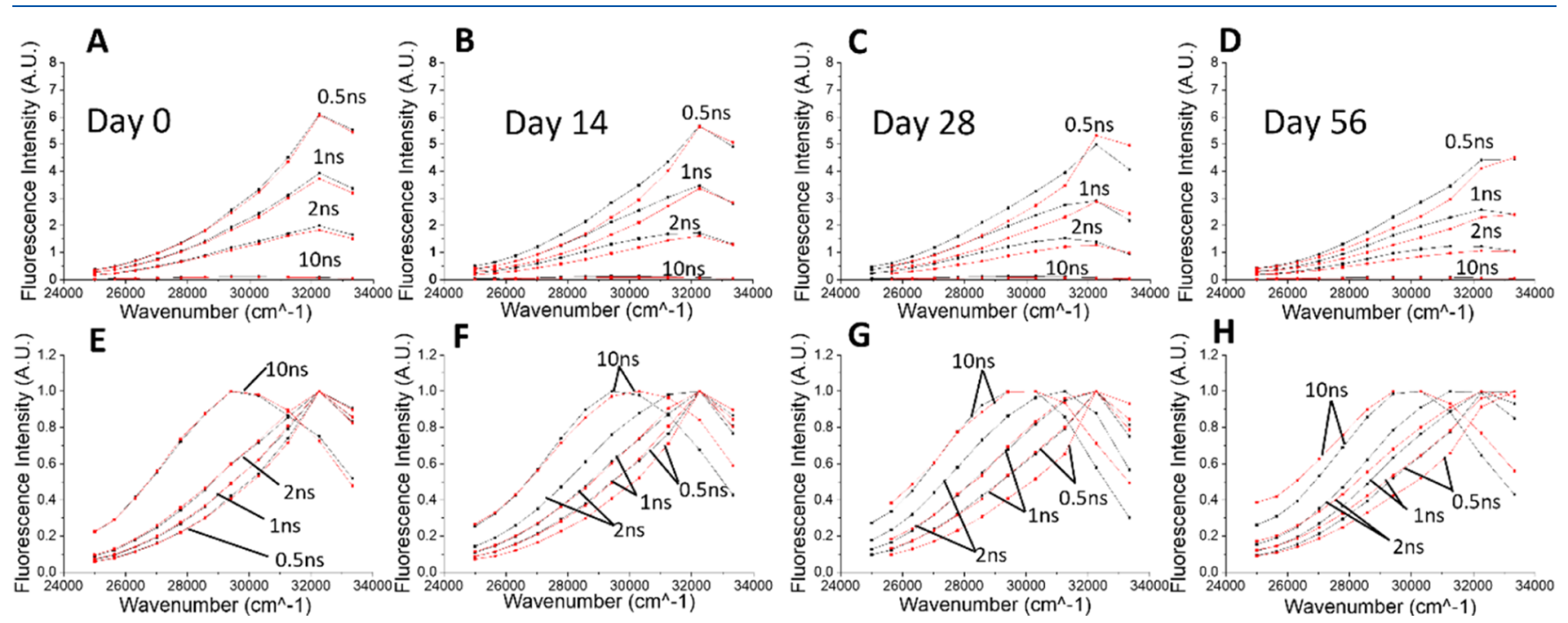

Figure 3. TRES spectra of free collagen (black) and collagen-glucose (red) on day 0 (A), day 14 (B), day 28 (C), and day 56 (D). Plots (E-H) show the same spectra after normalization. 


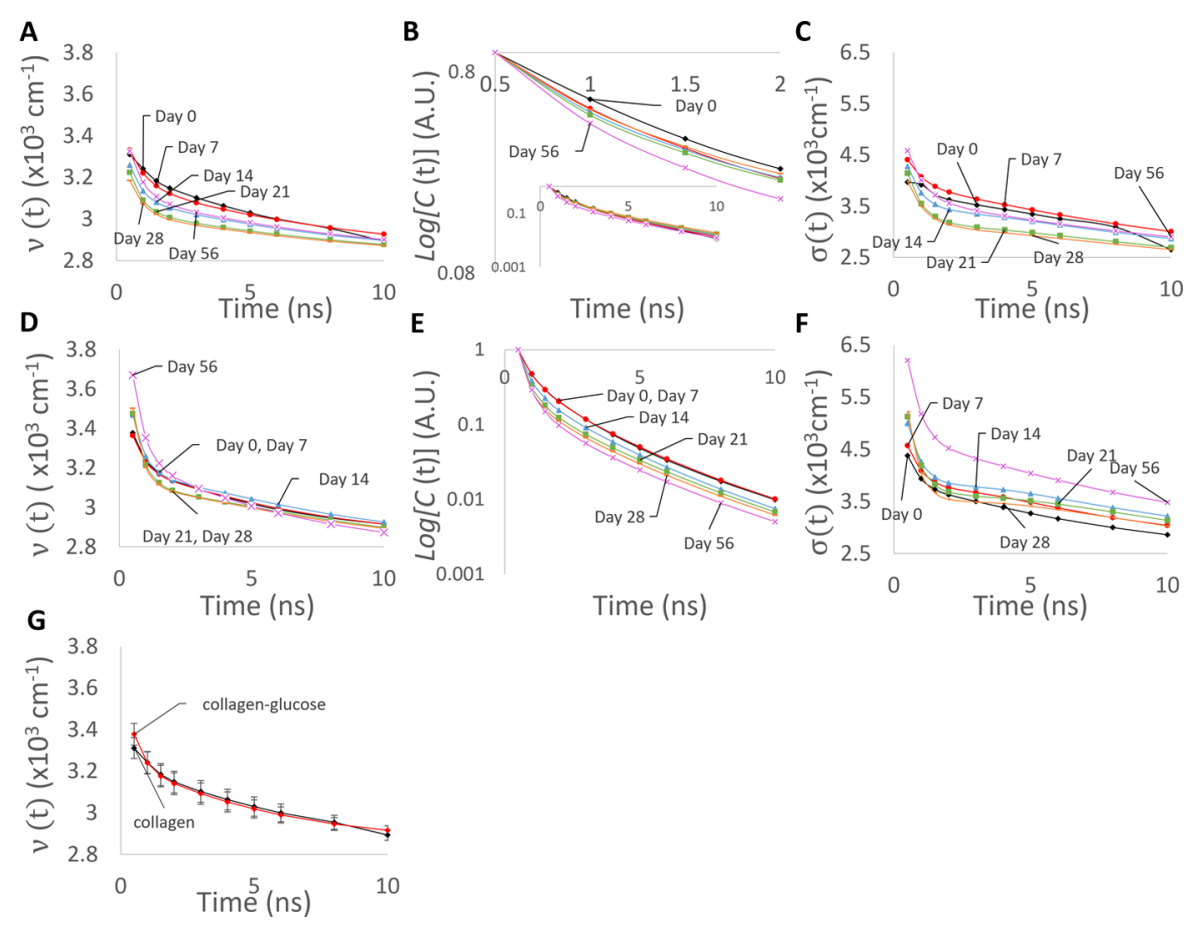

Figure 4. Plots (A,D) show the time evolution of the peak position $v(t)$ of the emitting fluorophores for collagen and collagen-glucose, respectively, excited at $280 \mathrm{~nm}$. The time evolution of fluorescence intensity $\mathrm{C}(t)$ is shown for collagen and collagen-glucose in plots (B,E), with each spectra normalized. The inset shows the same data over a longer time range. The six lines show day 0 , day 7 , day 14 , day 21 , day 28 , and day 56. Plots $(\mathrm{C}, \mathrm{F})$ show the changes in $\sigma$ parameters (see eq 5). Part (G) shows $(t)$ for collagen and collagen-glucose on day 0 for comparison, with the error bars showing the $90 \%$ confidence intervals for the fitted values.

fluorescence spectra shown in Figure 1B. Moreover, Figure $4 \mathrm{~B}$,E shows that the normalized fluorescence decays $C(t)$ of tyrosine in collagen and collagen-glucose samples, do not change substantially. Figure 4C,F presents falls in $\sigma(t)$ factors occurring on the ns time scale, indicating that the spectrum becomes narrower at the later times after excitation. We explain this observation by the existence of the collagen aggregates characterized by a continuous and narrow distribution of spectral peaks and dielectric relaxation times, rather than two or more distinct aggregates, which would lead to the optimal $M=2$ or more in data analysis.

To conclude this section, the broader Tyr steady-state fluorescence spectra and the systematic red shift of the TRES in the sample of free collagen suggest collagen aggregation, which leads to increasing volumes of the individual aggregates. Moreover, the narrower steady-state spectra and reduced changes in the spectral shifts observed in the presence of glycation indicates that the collagen aggregation is limited by glucose. However, glucose does not affect the fluorescence decays of tyrosines.

Pepsin-Digestible Cross-Link Responses (Excitation $340 \mathrm{~nm})$. The fluorescence responses of pepsin-digestible cross-links in collagen were studied by exciting the collagen and collagen-glucose samples at $340 \mathrm{~nm}$. Figure 5A shows how the steady-state fluorescence spectrum of each sample evolves over time. Free collagen initially has the fluorescence peak at $\sim 405 \mathrm{~nm}$. The intensity of the spectrum then increases, mostly during the first 7 days, and undergoes a red spectral shift up to $\sim 425 \mathrm{~nm}$ on day 35 .

The fluorescence spectrum of the collagen-glucose sample increases steadily over the 35 days. It reaches a higher value and is broader than the spectrum of the free collagen sample.
Figure 5B allows for the comparison of the fluorescence emission of free collagen and collagen-glucose samples over 35 days, with both spectra normalized. From day 0 , the sample with glucose shows a broader spectrum, and there is also a difference in the wavelength of peak fluorescence: $\sim 405 \mathrm{~nm}$ for free collagen, which moves gradually to 425 , and $\sim 425 \mathrm{~nm}$ for collagen-glucose, which remains stable. We attribute the emission in the $400-430 \mathrm{~nm}$ range to the formation of dityrosine from the neighboring tyrosine residues ${ }^{34}$ and this process occurs in both the free collagen and glucose-collagen samples. However, the further increase in the fluorescence intensity and the shift of the spectra observed in the collagenglucose sample only is likely to be an indication of multiple cross-links formed between glycated collagen molecules. ${ }^{14,16}$

To gain further information, TRES measurements were performed at various time points after sample preparation, at detection wavelengths from $390-500 \mathrm{~nm}$. The absolute and normalized TRES for collagen and collagen-glucose are presented in Figure 6.

The TRES of free collagen (black curves) on day 0 show no change in their shape during the decay (see the plot of normalized spectra, black curves in Figure $6 \mathrm{E}$ ), indicating no ns time scale processes. The broad peak of the spectrum at the range $\sim 24,700$ and $\sim 23,300 \mathrm{~cm}^{-1}$, that is, $405-429 \mathrm{~nm}$, is consistent with the steady-state spectra. The measurements performed at later days show a peak at $\sim 21,100 \mathrm{~cm}^{-1}$, that is, $475 \mathrm{~nm}$, and the gradual transition of the fluorescence peak toward the red on a nanosecond time scale.

In the sample containing glucose (red spectra), TRES data show a similar transition; however, the emission peak at 475 $\mathrm{nm}$ is already apparent at day 0 , which may suggest that glucose accelerates the formation of this fluorescent residue. 

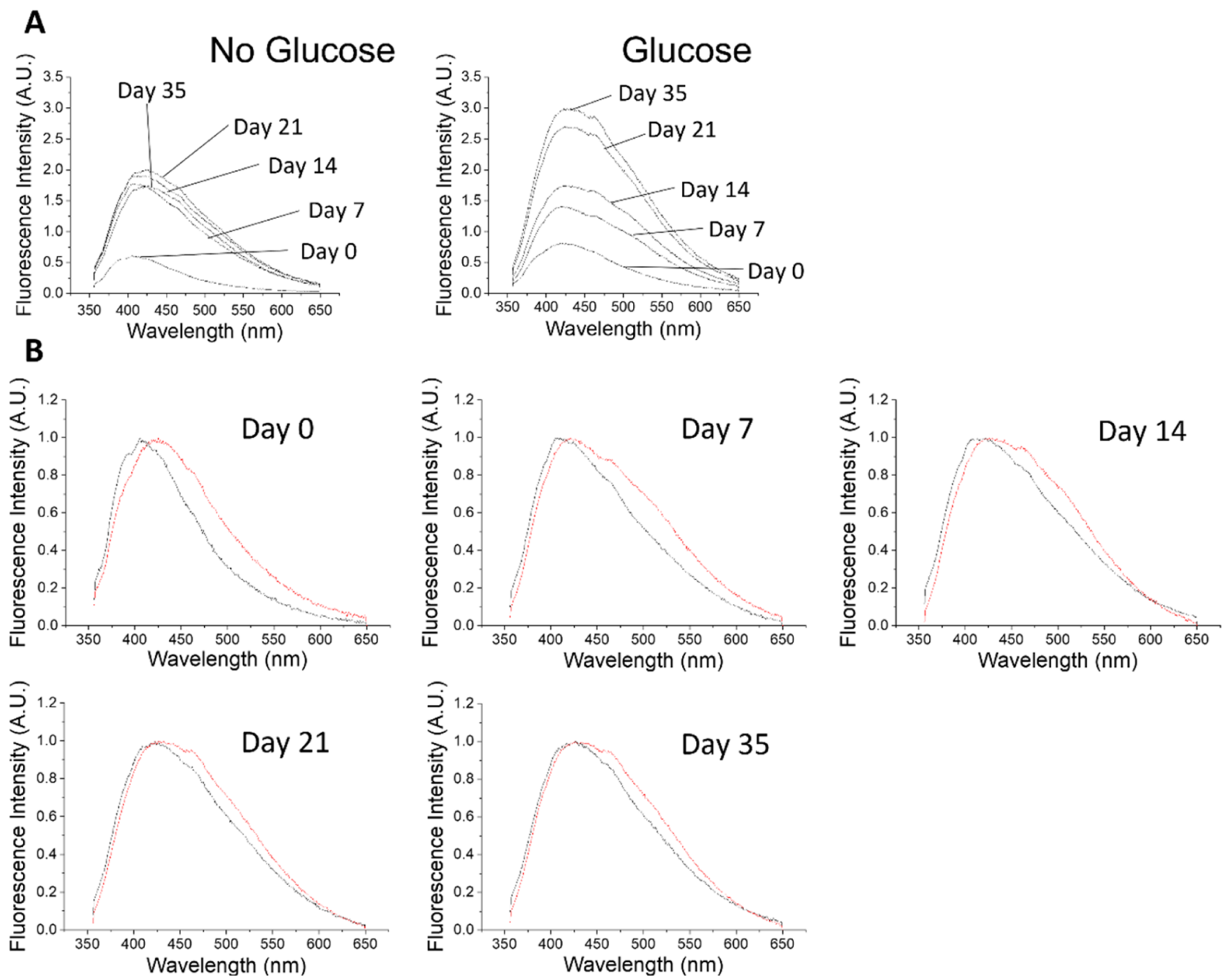

Figure 5. Fluorescence emission spectra for free collagen and collagen glucose when excited at $340 \mathrm{~nm}$. (A) Fluorescence changes over time for collagen and collagen-glucose at days $0,7,14,21$, and 35. (B) Collagen (black) and collagen-glucose (red) on days 0, 7, 14, 21, and 35, with both samples spectra normalized to a peak fluorescence.

The direct inspection of TRES suggests at least two fluorescent residues.

Indeed, although the analysis of the Akaike information criterion (see Table T2) again requires the consideration of a one-component Toptygin model $(M=1)$, the analysis of the evolution of the $v(t)$ parameter is characteristic for multicomponent kinetics (Figure 7), which we explain below.

The initial (Day 0) peak position of the free collagen spectrum starts at $\sim 22,500 \mathrm{~cm}^{-1}$ (Figure $7 \mathrm{~A}$ ) and at $\sim 22,000$ $\mathrm{cm}^{-1}$ for collagen-glucose (Figure $7 \mathrm{C}$ ) and they behave differently, see Figure 7E for comparison. There is no shift in $v(t)$ following excitation for free collagen at day 0 ; however, the measurements taken on the later days show a substantial drop of the initial $v(t)$ to $\sim 20,900 \mathrm{~cm}^{-1}$. All $v(t)$ dependencies show a further red shift during the first $2 \mathrm{~ns}$, and then the $v(t)$ plots start to increase (which is most visible for the data obtained on day 7). Again, this observation contradicts the homogeneous dielectric relaxation process, where the monotonic red shift is expected. It can only be explained by the fluorescence of a number of species of individual fluorescence lifetimes, dielectric relaxation rates, and so forth. Obtaining $M$ $=1$ in the Akaike analysis can be again explained by the presence of aggregates characterized by a distribution of fluorescence characteristics, rather than a small number of aggregates of distinctively different parameters.

The $v(t)$ for the collagen-glucose sample on day 0 drops from 22,000 to $\sim 20,500 \mathrm{~cm}^{-1}$ during the first $2 \mathrm{~ns}$. In the case of older samples, the starting value of $v(t)$ is $\sim 20,600 \mathrm{~cm}^{-1}$ which is also followed by a similar red shift within the first $2 \mathrm{~ns}$. This behavior of $v(t)$ demonstrates that in the presence of glucose, the formation of cross-links occurs differently, leading to only one type of the fluorescent cross-link.

The $\log (C(t))$ curves (Figure 7B,D) seem to confirm this observation. The changes in the rates of the decays on different days in the pure collagen sample (Figure 7B) are not monotonic. Indeed, the decay in Day 7 is faster than in Day 0 , and then it slows down again at the later days. This suggests the presence of multiple fluorescent cross-links whose contributions change over time. The decays observed in the glycated sample (Figure 7D), however, show a stable trend of decreasing decay rates, which is likely to be observed when there is a single or a set of uniform fluorescent residues.

To conclude, in a pure collagen sample, more than one type of fluorescent cross-link is formed in the 35 day period, while 

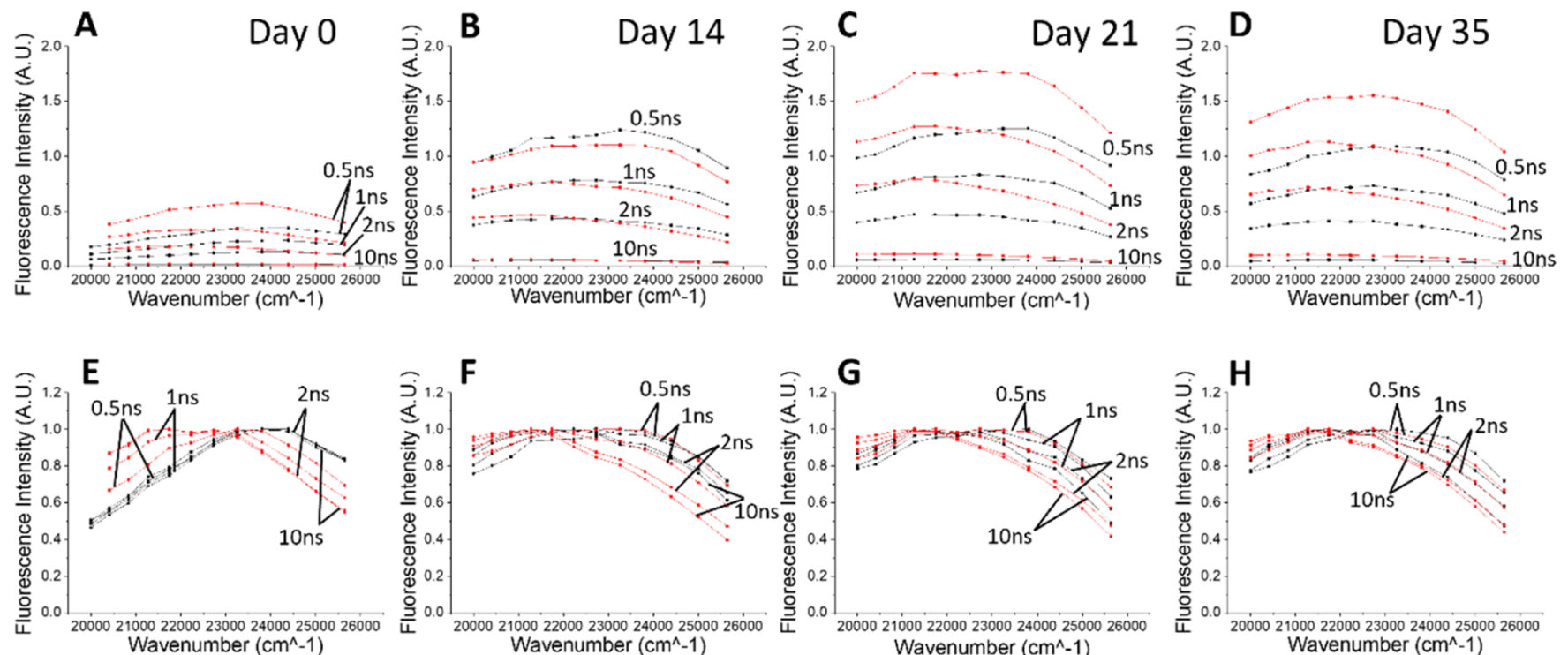

Figure 6. Examples of TRES spectra of free collagen (black) and collagen-glucose (red) on day 0 (A), day 14 (B), day 21 (C), and day 35 (D). Plots $(\mathrm{E}-\mathrm{H})$ show the same spectra after normalization.

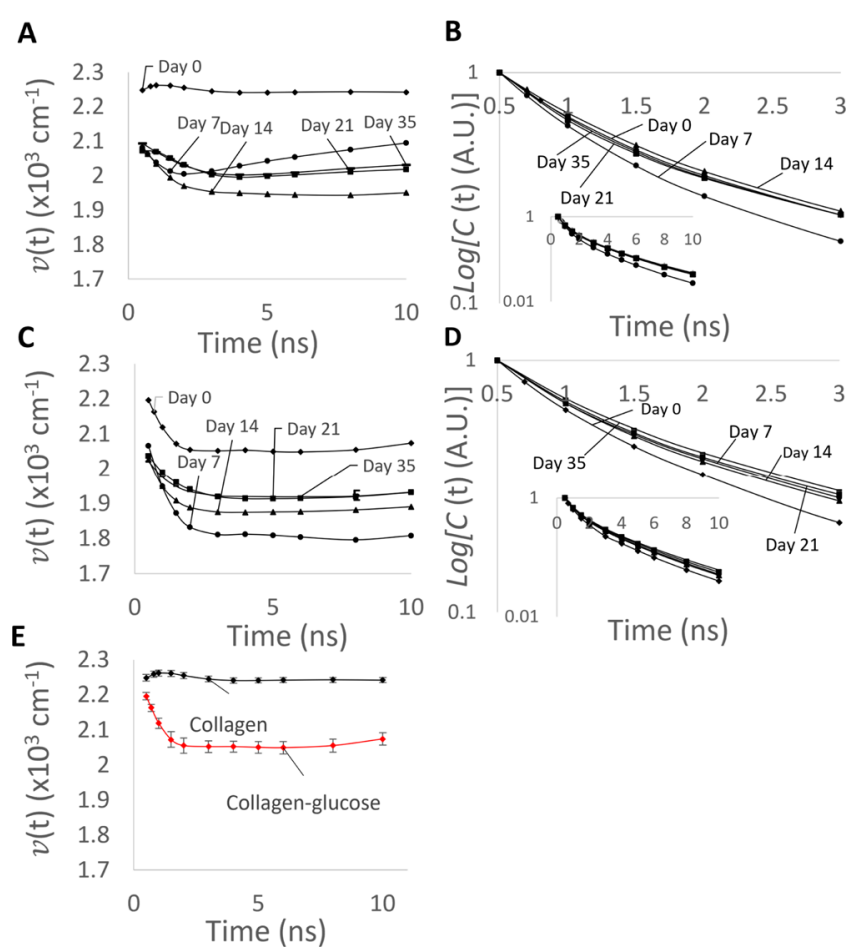

Figure 7. Plots $(\mathrm{A}, \mathrm{C})$ show the time evolution of the peak position $v(t)$ of the emitting fluorophores for collagen and collagen-glucose, respectively, excited at $340 \mathrm{~nm}$. The normalized time evolution of the fluorescence intensity $\mathrm{C}(t)$ is shown for collagen and collagenglucose in plots $(\mathrm{B}, \mathrm{D})$. The inset shows the same data over a longer timer range. The five lines show day 0 , day 7 , day 14 , day 21 , and day 35. Part (E) shows $(t)$ for collagen and collagen-glucose on day 0 for comparison, with the error bars showing the $90 \%$ confidence intervals for the fitted values.

in the collagen-glucose sample, a single fluorescent form is created, demonstrating a substantial impact of glycation on collagen aggregation.

\section{CONCLUSIONS}

The effects of glucose on collagen's intrinsic fluorescence has been studied using a TRES-based technique. Our aim was to identify the characteristic features of the excited-state kinetics occurring in the glucose-triggered aggregation of collagen. Intrinsic fluorescence responses were collected for the excitation wavelengths 280 and $340 \mathrm{~nm}$ to observe changes in the fluorescence of intrinsic tyrosine and fluorescent crosslinks.

It has been found that glucose does not have a substantial impact on the fluorescence of intrinsic Tyr (excitation at 280 $\mathrm{nm}$ ). Indeed, as glucose binds to collagen at lysine residues, and lysine makes up only $3.9 \%$ of collagen, ${ }^{15,35}$ formation of lysine-glucose complexes is not likely to impact Tyr's local microenvironment. Nevertheless, the TRES of both free collagen and collagen-glucose samples excited at $280 \mathrm{~nm}$ do change over time, which is due to protein aggregation.

Glucose, however, does have a substantial influence on the formation of pepsin-digestible collagen cross-links emitting at $\lambda$ $>400 \mathrm{~nm}$ (excitation at $340 \mathrm{~nm}$ ). The observed changes in TRES show the impact of glucose on the formation pathways of new cross-links and protein aggregates.

However, as the TRES approach that we have used does not assume any specific model of the underlying excited state kinetics; further studies will be required to identify the best fit model of the kinetics and for determining the molecular mechanisms involved.

\section{ASSOCIATED CONTENT}

\section{Supporting Information}

The Supporting Information is available free of charge at https://pubs.acs.org/doi/10.1021/acs.jpcb.1c05001.

Examples of raw TCSPC data analysis, calculated TRES values versus.TRES model curves, $\mathrm{AIC}_{\mathrm{c}}$ definition, and examples of calculated values (PDF) 


\section{AUTHOR INFORMATION}

Corresponding Author

Olaf J. Rolinski - Photophysics Group, Department of Physics, University of Strathclyde, Glasgow G4 ONG, U.K.; 이이.org/0000-0002-7838-779X; Email: o.j.rolinksi@ strath.ac.uk

\section{Authors}

Rhona Muir - Photophysics Group, Department of Physics, University of Strathclyde, Glasgow G4 ONG, U.K.

Shareen Forbes - BHF Centre for Cardiovascular Science, The Queen's Medical Research Institute, University of Edinburgh, Edinburgh EH16 4TJ, U.K.

David J.S. Birch - Photophysics Group, Department of Physics, University of Strathclyde, Glasgow G4 ONG, U.K.

Vladislav Vyshemirsky - School of Mathematics and Statistics, University of Glasgow, Glasgow G12 8QQ U.K.

Complete contact information is available at: https://pubs.acs.org/10.1021/acs.jpcb.1c05001

\section{Author Contributions}

R.M. performed the measurements, data fitting, and wrote first draft of the manuscript, S.F. provided support on biomedical aspects, D.J.S.B. supported data interpretation and corrections to the manuscript, V.V. created and supported the use of software that considers the scattered light in the decays, O.J.R. led the research, data interpretation, and the preparation of the final manuscript.

Notes

The authors declare no competing financial interest.

\section{ACKNOWLEDGMENTS}

R.M. would like to thank OPTIMA-The EPSRC and MRC Centrer for Doctoral Training in Optical Medical Imaging for her $\mathrm{PhD}$ studentship.

\section{REFERENCES}

(1) Nathan, D. M.; Edic, D. The Diabetes Control and Complications Trial/Epidemiology of Diabetes Interventions and Complications Study at 30 Years: Overview. Diabetes Care 2014, 37, 9-16.

(2) World Health Organization. Global Report on Diabetes, 2016; vol 978.

(3) Singh, V. P.; Bali, A.; Singh, N.; Jaggi, A. S. Advanced glycation end products and diabetic complications. Korean J. Physiol. Pharmacol. 2014, 18, 1-14.

(4) Negre-Salvayre, A.; Salvayre, R.; Augé, N.; Pamplona, R.; Portero-Otín, M. Hyperglycemia and Glycation in Diabetic Complications. Antioxid. Redox Signal 2009, 11, 3071-3109.

(5) Avery, N. C.; Bailey, A. J. The effects of the Maillard reaction on the physical properties and cell interactions of collagen. Pathol. Biol. 2006, 54, 387-395.

(6) Verzijl, N.; DeGroot, J.; Oldehinkel, E.; Bank, R. A.; Thorpe, S. R.; Baynes, J. W.; Bayliss, M. T.; Bijlsma, J. W. J.; Lafeber, F. P. J. G.; Tekoppele, J. M. Age-related accumulation of Maillard reaction products in human articular cartilage collagen. Biochem. J. 2000, 350, 381-387.

(7) Reigle, K. L.; Di Lullo, G.; Turner, K. R.; Last, J. A.; Chervoneva, I.; Birk, D. E.; Funderburgh, J. L.; Elrod, E.; Germann, M. W.; Surber, C.; et al. Non-enzymatic glycation of type I collagen diminishes collagen- proteoglycan binding and weakens cell adhesion. J. Cell. Biochem. 2008, 104, 1684-1698.

(8) Usha, R.; Jaimohan, S. M.; Rajaram, A.; Mandal, A. B. Aggregation and self assembly of non-enzymatic glycation of collagen in the presence of amino guanidine and aspirin: An in vitro study. Int. J. Biol. Macromol. 2010, 47, 402-409.

(9) Cooper, M.E.; Bonnet, F.; Oldfield, M.; Jandeleit-dahm, K. Mechanisms of diabetic vasculopathy: an overview. Am. J. Hypertens. 2001, 14, 475-486.

(10) Yuen, A.; Laschinger, C.; Talior, I.; Lee, W.; Chan, M.; Birek, J.; Young, E. W. K.; Sivagurunathan, K.; Won, E.; Simmons, C. A.; et al. Methylglyoxal-modified collagen promotes myofibroblast differentiation. Matrix Biol. 2010, 29, 537-548.

(11) The DCCT Research Group. The Effect of Intensive Treatment of Diabetes on the Development and Progression of Long-Term Complications in Insulin-Dependent Diabetes Mellitus. N. Engl. J. Med. 1993, 329, 977-986.

(12) Makita, Z.; Radoff, S.; Rayfield, E. J.; Yang, Z.; Skolnik, E.; Delaney, V.; Friedman, E. A.; Cerami, A.; Vlassara, H. Advanced glycosylation end products in patients with diabetic nephropathy. $\mathrm{N}$. Engl. J. Med. 1991, 325, 836-842.

(13) Genuth, S.; Sun, W.; Cleary, P.; Sell, D. R.; Dahms, W.; Malone, J.; Sivitz, W.; Monnier, V. M. Glycation and carboxymethyllysine levels in skin collagen predict the risk of future 10-year progression of diabetic retinopathy and nephropathy in the diabetes control and complications trial and epidemiology of diabetes interventions and complications p. Diabetes 2005, 54, 3103-3111.

(14) Fokkens, B. T.; Smit, A. J. Skin fluorescence as a clinical tool for non-invasive assessment of advanced glycation and long-term complications of diabetes. Glycoconj. J. 2016, 33, 527-535.

(15) Hori, M.; Yagi, M.; Nomoto, K.; Ichijo, R.; Shimode, A.; Kitano, T.; Yonei, Y. Experimental Models for Advanced Glycation End Product Formation Using Albumin, Collagen, Elastin, Keratin and Proteoglycan. Anti Aging Med. 2012, 9, 125-134.

(16) Brennan, M. Changes in Solubility, Non-enzymatic Glycation, and Fluorescence of Collagen in Tail Tendons from Diabetic Rats*. J. Biol. Chem. 1989, 264, 20947-20952.

(17) Kollias, N.; Gillies, R.; Moran, M.; Kochevar, I. E.; Anderson, R. R. Endogenous Skin Fluorescence In Vivo on Human Skin. J. Invest. Dermatol. 1998, 111, 776-780.

(18) Lakowicz, J. R. Principles of Fluorescence Spectroscopy; Springer, 2006.

(19) Nguyen, T. T.; Gobinet, C.; Feru, J.; Pasco, S. B.; Manfait, M.; Piot, O. Characterization of Type I and IV Collagens by Raman Microspectroscopy: Identi fi cation of Spectral Markers of the DermoEpidermal Junction. Spectrosc. Int. J. 2012, 27, 421-427.

(20) Teale, F. W. J.; Weber, G. Ultraviolet Fluorescence of the Aromatic Amino Acids. Biochem. J. 1957, 65, 476-482.

(21) Nuytinck, L.; Tükel, T.; Kayserili, H.; Apak, M. Y.; De Paepe, A. Glycine to tryptophan substitution in type I collagen in a patient with OI type III: a unique collagen mutation. J. Med. Genet. 2000, 37, $371-375$.

(22) Shen, Y.; Zhu, D.; Lu, W.; Liu, B.; Li, Y.; Cao, S. The Characteristics of Intrinsic Fluorescence of Type I Collagen Influenced by Collagenase I. Appl. Sci. 2018, 8, 1947.

(23) Kollias, N.; Gillies, R.; Moran, M.; Kochevar, I. E.; Anderson, R. R. Endogenous Skin Fluorescence Includes Bands that may Serve as Quantitative Markers of Aging and Photoaging. J. Invest. Dermatol. 1998, 111, 776-780.

(24) Na, R.; Stender, I.-M.; Henriksen, M.; Wulf, H. C. Autofluorescence of human skin is age-related after correction for skin pigmentation and redness. J. Invest. Dermatol. 2001, 116, 536540 .

(25) Meerwaldt, R.; Graaff, R.; Oomen, P. H. N.; Links, T. P.; Jager, J. J.; Alderson, N. L.; Thorpe, S. R.; Baynes, J. W.; Gans, R. O. B.; Smit, A. J. Simple non-invasive assessment of advanced glycation endproduct accumulation. Diabetologia 2004, 47, 1324-1330.

(26) Meerwaldt, R.; Hartog, J. W. L.; Graaff, R.; Huisman, R. J.; Links, T. P.; den Hollander, N. C.; Thorpe, S. R.; Baynes, J. W.; Navis, G.; Gans, R. O. B.; et al. Skin Autofluorescence , a Measure of Cumulative Metabolic Stress and Advanced Glycation End Products, Predicts Mortality in Hemodialysis Patients. J. Am. Soc. Nephrol. 2005, 16, 3687-3693. 
(27) Easter, J. H.; DeToma, R. P.; Brand, L. Nanosecond timeresolved emission spectroscopy of a fluorescence probe adsorbed to L-alpha-egg lecithin vesicles. Biophys. J. 1976, 16, 571-583.

(28) Alghamdi, A.; Vyshemirsky, V.; Birch, D. J. S.; Rolinksi, O. J. Detecting beta-amyloid aggregation from time-resolved emission spectra. Methods Appl. Fluoresc. 2018, 6, 024002.

(29) Chung, L. H. C.; Birch, D. J. S.; Vyshemirsky, V.; Ryadnov, M. G.; Rolinksi, O. J. Insulin aggregation tracked by its intrinsic TRES. Appl. Phys. Lett. 2017, 111, 263701.

(30) Rolinski, O. J.; Wellbrock, T.; Birch, D. J. S.; Vyshemirsky, V. Tyrosine Photophysics During the Early Stages of $\beta$ - Amyloid Aggregation Leading to Alzheimer's. J. Phys. Chem. Lett. 2015, 6, $3116-3120$

(31) Toptygin, D.; Brand, L. Spectrally- and time-resolved fluorescence emission of indole during solvent relaxation: a quantitative model. Chem. Phys. Lett. 2000, 322, 496-502.

(32) Rolinski, O. J.; Mclaughlin, D.; Birch, D. J. S.; Vyshemirsky, V. Resolving environmental microheterogeneity and dielectric relaxation in fluorescence kinetics of protein. Methods Appl. Fluoresc. 2016, 4, 024001.

(33) Burnham, K. P.; Anderson, D. R. Model Selection and Multimodel Inference, A Practical Information-Theoretic Approach; Springer, 2002.

(34) Menter, J. M. Temperature dependence of collagen fluorescence. Photochem. Photobiol. Sci. 2006, 5, 403-410.

(35) Bolboac, S. D.; Jäntschi, L. Amino Acids Sequence Analysis on Collagen. Bull. USAMV-CN 2007, 63, 311-316.

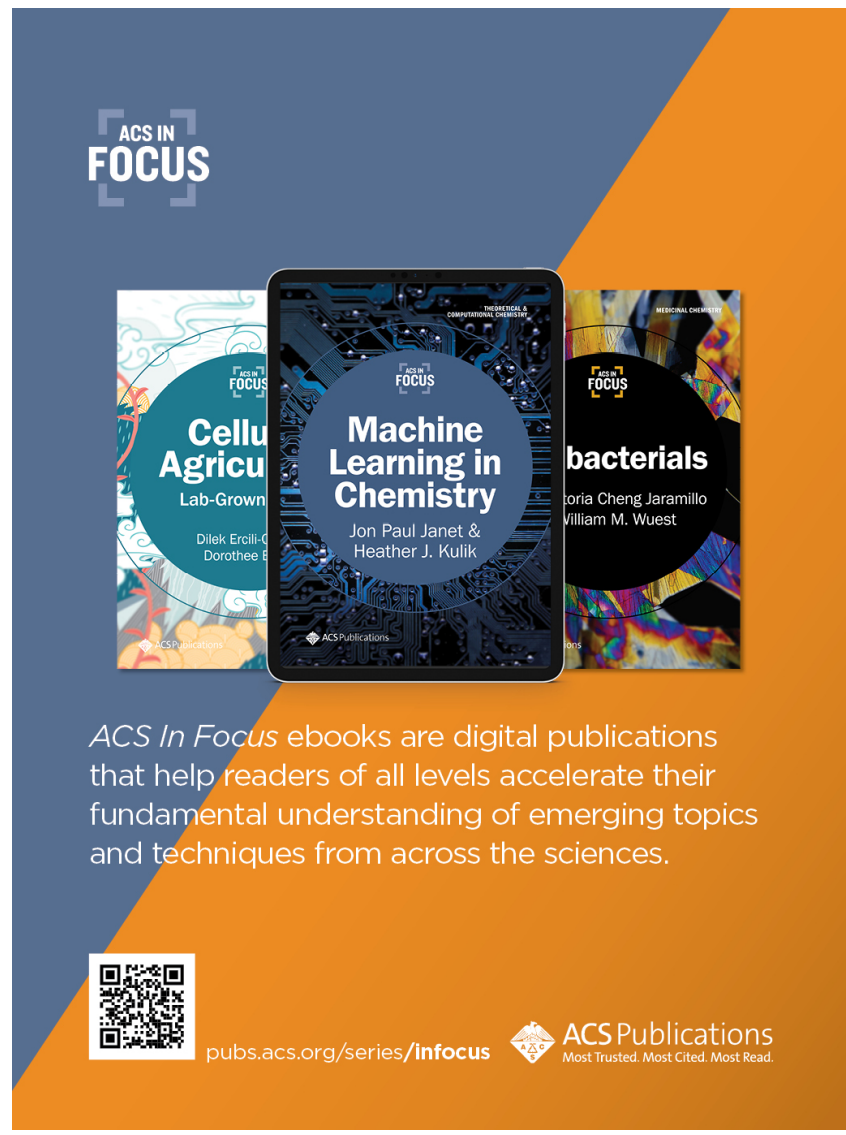

